\title{
Non-Orthogonal Multiple Access and Carrierless Amplitude Phase Modulation for 5G
} Mobile Networks

Altabas, Jose A. ; Rommel, Simon; Puerta Ramírez, Rafael; Izquierdo, David ; Garces, Ignacio; Lazaro, Jose A.; Vegas Olmos, Juan José; Tafur Monroy, Idelfonso

Published in:

2017 European Conference on Optical Communication (ECOC)

Link to article, DOI:

10.1109/ECOC.2017.8346106

Publication date:

2017

Document Version

Peer reviewed version

Link back to DTU Orbit

Citation (APA):

Altabas, J. A., Rommel, S., Puerta Ramírez, R., Izquierdo, D., Garces, I., Lazaro, J. A., Vegas Olmos, J. J., \& Tafur Monroy, I. (2017). Non-Orthogonal Multiple Access and Carrierless Amplitude Phase Modulation for $5 \mathrm{G}$ Mobile Networks. In 2017 European Conference on Optical Communication (ECOC) IEEE.

https://doi.org/10.1109/ECOC.2017.8346106

\section{General rights}

Copyright and moral rights for the publications made accessible in the public portal are retained by the authors and/or other copyright owners and it is a condition of accessing publications that users recognise and abide by the legal requirements associated with these rights.

- Users may download and print one copy of any publication from the public portal for the purpose of private study or research.

- You may not further distribute the material or use it for any profit-making activity or commercial gain

- You may freely distribute the URL identifying the publication in the public portal 


\title{
Non-Orthogonal Multiple Access and Carrierless Amplitude Phase Modulation for 5G Mobile Networks
}

\author{
Jose A. Altabas(1), Simon Rommel(2), Rafael Puerta(2), David Izquierdo(1,3), Ignacio Garces(1), \\ Jose A. Lazaro(4), Juan Jose Vegas Olmos( ${ }^{(5)}$, Idelfonso Tafur Monroy(2)
}

\author{
(1) Aragon Institute of Engineering Research (I3A), Universidad de Zaragoza, Zaragoza, Spain; email: \\ jaltabas@unizar.es \\ (2) Department of Photonics Engineering, Technical University of Denmark (DTU), Kgs. Lyngby, \\ Denmark, email: sirem@fotonik.dtu.dk \\ (3) Centro Universitario de la Defensa (CUD), Zaragoza, Spain \\ (4) Universitat Politecnica de Catalunya (UPC-BarcelonaTech), Barcelona, Spain \\ (5) Mellanox Technologies, Ledreborg Alle 130B, 4000, Roskilde, Denmark
}

\begin{abstract}
A$ combined NOMA and multiCAP scheme is proposed for capacity enhancement of $5 \mathrm{G}$ mobile networks and experimentally tested over a $W$-band millimeter-wave radio-over-fiber system. The evaluated NOMA-CAP system provides an aggregated transmission rate of $30 \mathrm{Gbps}$.
\end{abstract}

\section{Introduction}

Traffic demand over wireless networks is growing exponentially due to new multimedia streaming services, the Internet of Things (IoT) and machine-to-machine communications ${ }^{1}$. This growing traffic on $5 \mathrm{G}$ networks requires the use of non-congested wireless bands such as millimeter-wave (mm-wave), and so it will require a re-design of front- and backhaul architectures for radio access networks (RANs). The use of centralized radio access networks (C-RANs) is suggested as a key enabler ${ }^{1,2}$ and its combination with radio-over-fiber (RoF) on passive optical networks (PON) is a promising candidate to flexibly support $5 \mathrm{G}$ mobile networks ${ }^{2}$.

In RANs, the design of the access to the medium is a key technology in order to improve system capacity and to dynamically allocate the available resources. Non-orthogonal multiple access (NOMA) is a promising candidate for addressing these requirements and to enhance both capacity and flexibility of the network ${ }^{3}$.

The NOMA technique multiplexes the data of several users in the power domain by adding the contributing signals and allows combination with time- or frequency multiplexing. Successive interference cancellation (SIC) is employed in the terminal units in order to recover the contributing signals and thus demultiplex the NOMA users ${ }^{3}$.

High data rate demands require a migration from inefficient modulation formats, such as impulse radio or on-off keying, to advanced modulation schemes as multi-band carrierless amplitude phase (multiCAP) modulation ${ }^{4}$.

In this paper, NOMA and multiCAP techniques are combined in order to allow flexible resource provisioning that addresses the dynamic nature of user density and capacity demands. Fig. 1 shows a possible application scenario where two users are located at different distances to the base station (BS) and share the medium by NOMA multiplexing.

The NOMA-CAP is experimentally validated on a W-band RoF link. NOMA-CAP using six $1.25 \mathrm{GHz}$ multiCAP bands and two quadrature phase shift keying (QPSK) NOMA levels has been tested achieving aggregated transmission rate of $30 \mathrm{Gbps}$.

\section{Experimental Setup}

Figs. 2 and Fig. 3 shows the transmitter and receiver digital signal processing (DSP) respectively, while Fig. 4 shows the experimental setup used to validate the proposed NOMA-CAP transmission over a hybrid photonic-wireless link.

The transmitter DSP for NOMA-CAP, shown in Fig. 2, distributes the data of each user or NOMA level among all the multiCAP bands and maps them to QPSK symbols. The NOMA levels are power weighted and added for each multiCAP band. The band signals are up-sampled and filtered with a pair of band specific multiCAP orthogonal filters. Finally, the multiCAP bands are aggregated. In the test case, two NOMA levels and six $1.25 \mathrm{GHz}$ multiCAP bands are used.

The optical signal generation, depicted in Fig. 4a), is based on the modulation of a narrow linewidth external cavity laser (ECL) at $\lambda=1550 \mathrm{~nm}$ by a Mach-Zehnder modulator (MZM) biased at its minimum transmission point with a sinusoidal signal at $f_{\mathrm{RF}} / 2$ from a vector signal generator (VSG). This configuration generates two

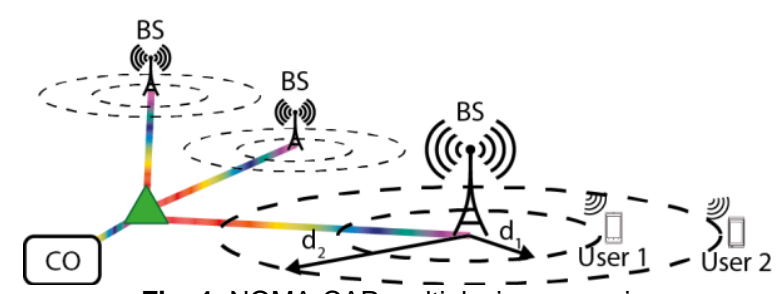

Fig. 1: NOMA-CĀP multiplexing scenario. 


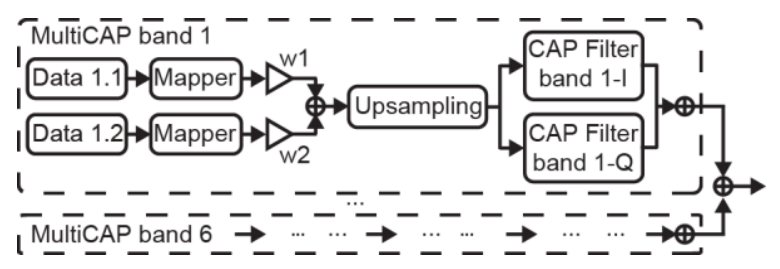

Fig. 2: Transmitter DSP block diagram.

spectral lines spaced at $f_{R F}=84 G H z$, which later serve for the photonic generation of the RF signal. After amplification in an erbium doped fiber amplifier (EDFA), the lines are separated in an arrayed waveguide grating (AWGG). Finally, one of the spectral lines is modulated with the NOMA-CAP signal using a second MZM. The power of both lines are adjusted to be equal employing a variable optical amplifier (VOA) before they are added and amplified by a second EDFA. The launch power is adjusted with a second VOA before transmission through $10 \mathrm{~km}$ of ITU-T G.652 standard single mode fiber (SMF).

Optical to RF conversion, shown in Fig. 4b), is performed by a RAU designed for minimum complexity. The high-speed photodiode (PD) receives the optical signal and the generated RF signal is the beating of the two optical lines. The $\mathrm{PD}$ bandwidth is $90 \mathrm{GHz}$ and its responsivity is $0.5 \mathrm{~A} / \mathrm{W}$. RF power is amplified by a $10 \mathrm{~dB}$ medium power amplifier (MPA) up to a saturation output power of $12 \mathrm{dBm}$. A parabolic antenna with a gain of $48 \mathrm{dBi}$ is used to transmit the signal.

The wireless receiver, depicted in Fig. 4c), consists of an identical antenna after which the RF signal is amplified by a $20 \mathrm{~dB}$ low noise amplifier (LNA) before down-conversion to an intermediate frequency (IF) at $f_{I F}=f_{R F}-f_{L O}$ is performed in a balanced mixer. The local oscillator (LO) for the mixer is obtained from a passive frequency doubler, driven with a sinusoid at fLO/2 from a second VSG. The resulting IF signal is DC blocked and amplified before it is recorded on an oscilloscope (DSO) for offline processing.

The receiver DSP block diagram, which is the same for all users, is shown in Fig. 3a) and consists of a Costas loop for carrier frequency and -phase recovery for IF to baseband conversion, after the signal was band-filtered for noise bandwidth reduction. The baseband signal is lowpass filtered and each multiCAP band is extracted, employing the pair of orthogonal filters for

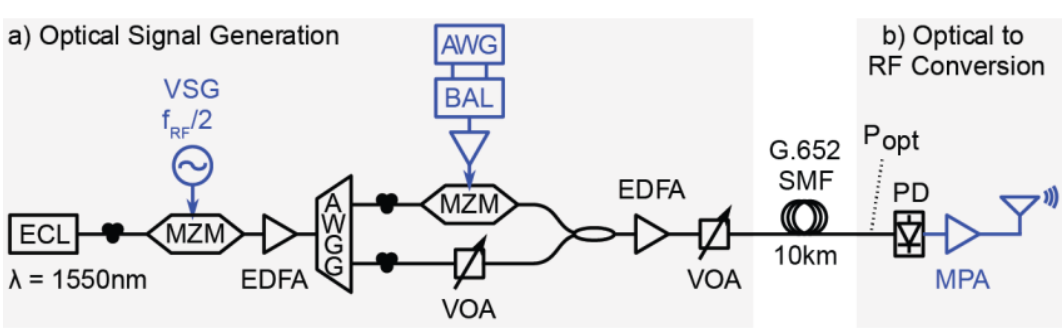

Fig. 4: Schematic of the experimental setup.

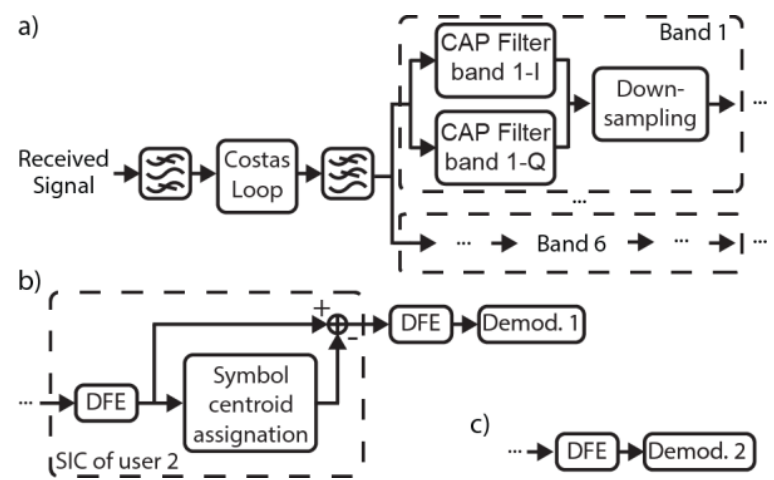

Fig. 3: Receiver DSP block diagram: (a) multiCAP band recovery (common to all receivers), (b) user 1 receiver with SIC processing, (c) user 2 receiver without SIC processing.

the band of interest. The close users have to implement SIC as is shown in Fig. 3b), consisting of a decision feedback equalizer (DFE) with 30 forward and 20 backward taps, calculation of the symbol centroid of the far user and subtraction from the equalized signal. After SIC, the DFE is applied again to the new signal and finally the signal is de-mapped. In the case of far users, shown in Fig. 3c), SIC is not required and the DFE and de-mapping are performed directly.

\section{Results}

The wireless transmission analysis for both user types with an available data rate of $15 \mathrm{Gbps}$ for each user, in terms of wireless distance and NOMA power ratio between users is shown in Fig. 5 (far user) and Fig. 6 (close user).

The received optical power on the PD is varied between $-3 \mathrm{~dB}$ and $+2.5 \mathrm{~dB}$ from a reference level of $0 \mathrm{dBm}$ using the second VOA, while wireless distance remains constant $(50 \mathrm{~m})$. This optical power is directly related to the transmitted RF power, so its variation can be used to emulate the wireless distance without moving the antennas? ${ }^{2}$. The total power of the NOMA-CAP signal is kept constant and the power ratio between users $\left(r_{\text {power }}\right)$ is defined as

$$
r_{\text {power }}=10 \log \left(\frac{w_{2}}{w_{1}}\right)
$$

where $w_{1}$ is the weight of the close user, while $w_{2}$ is the weight of the far user.

The distance analysis has been performed employing the respective BER limits of $3.8 \times 10^{-3}$ and $1.32 \times 10^{-2}$ for forward error corrections (FEC) with $7 \%$ and $25 \%$ overhead $(\mathrm{OH})^{5}$.

For user 2, i.e. the user located far, the NOMA

$$
\begin{array}{ll}
\text { b) Optical to } & \text { c) Wireless Receiver }
\end{array}
$$
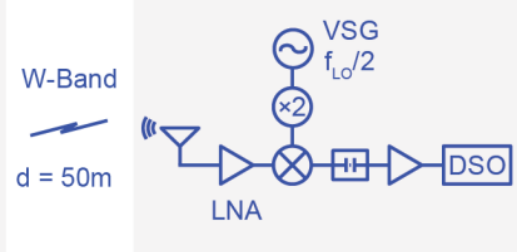


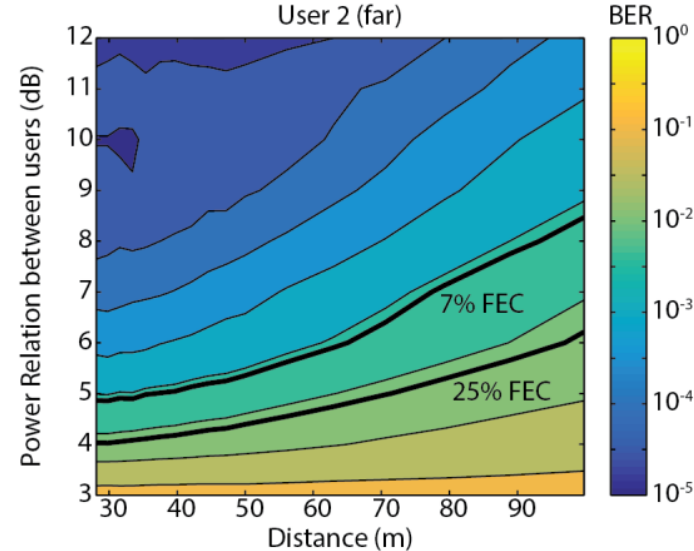

Fig. 5: BER map in terms of distance and power ratio between users for the user located far to the antenna.

multiplexing with the signal of user 1 will be regarded as an increment of the received noise and thus a reduction of the power ratio between users will increase the user BER and reduce the achievable distance for user 2 as seen Fig. 5 .

In the same scenario, user 1 , i.e. the user close by, will apply SIC cancellation before demodulation in order to remove the signal of user 2. Any errors in the calculation of the centroid of user 2 will propagate to the decoding of user 1 in the SIC process and thus they will cause a high BER for the user 1, as can be seen in Fig. 6 The use of a low power ratio between users, i.e. when the powers of user 1 and user 2 are comparable, will have a similar effect as placing user 1 at a long distance, resulting in errors in the calculation of the user 2 centroid and consequent error propagation to user 1 decoding. On the other hand, the BER of user 1 will increase with high power ratios as the signal will be too weak after SIC, even if the SIC process perfectly calculates the user 2 centroids. Thus, user 1 will only be successfully demodulated for intermediate power relations and for close distances, as seen in Fig. 6.

Tab. 1 summarizes the maximum reachable distances for both users under different power ratios. User 2 would thus prefer a scenario with high power ratio $\left(r_{\text {power }}>8 \mathrm{~dB}\right)$, and user 1 will prefer scenarios with medium power ratios $\left(r_{\text {powe }}=6-7 \mathrm{~dB}\right)$. The increment of the operational range of one user will decrease the range of the

Tab. 1: Summary of maximum achievable distances for both users and different power ratios. $x$ denotes no transmission distance possible below BER limit. $\varnothing$ denotes a distance greater than those experimentally tested.

\begin{tabular}{c|c|c|c|c} 
& \multicolumn{2}{|c|}{ User 1 (close) } & \multicolumn{2}{c}{ User 2 (far) } \\
\hline $\begin{array}{c}\text { Power } \\
\text { ratio }\end{array}$ & $7 \%$ & $25 \%$ & $7 \%$ & $25 \%$ \\
\hline $5 \mathrm{~dB}$ & $\mathrm{FEC}$ & FEC & FEC & FEC \\
\hline $6 \mathrm{~dB}$ & $43 \mathrm{~m}$ & $61 \mathrm{~m}$ & $36 \mathrm{~m}$ & $70 \mathrm{~m}$ \\
\hline $7 \mathrm{~dB}$ & $33 \mathrm{~m}$ & $59 \mathrm{~m}$ & $65 \mathrm{~m}$ & $96 \mathrm{~m}$ \\
\hline $8 \mathrm{~dB}$ & $\mathrm{x}$ & $52 \mathrm{~m}$ & $\varnothing$ & $\varnothing$ \\
\hline $9 \mathrm{~dB}$ & $\mathrm{x}$ & $40 \mathrm{~m}$ & $\varnothing$ & $\varnothing$
\end{tabular}

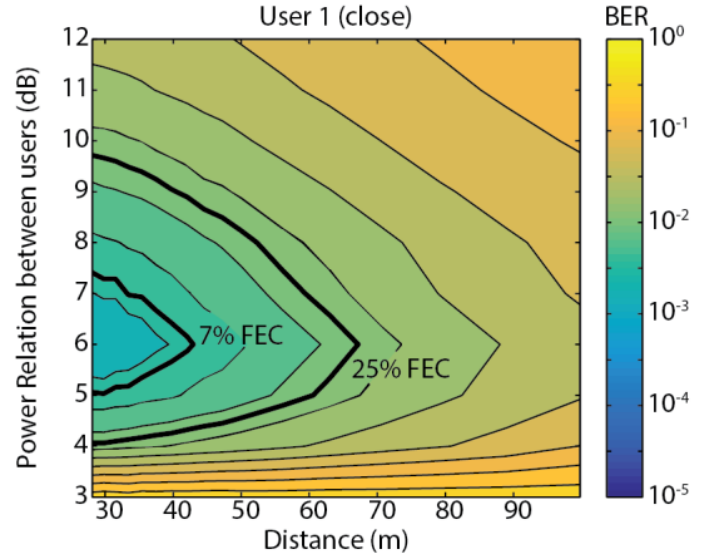

Fig. 6: BER map in terms of distance and power ratio between users for the user located close to the antenna.

other user. If the effective data rate can be reduced, a $25 \%$ OH FEC can be implemented and both users can be placed farther.

\section{Conclusions}

A combination of multiCAP and NOMA is proposed for application in future $5 \mathrm{G}$ mobile RANs, allowing optimization of capacity. An experimental demonstration was given for a NOMACAP RoF system in W-band.

The experimental demonstration achieved an aggregate system capacity of $30 \mathrm{Gbps}$ using a NOMA-CAP signal consisting of six $1.25 \mathrm{GHz}$ multiCAP and two NOMA levels. BER measurements are shown for different power ratios between the contributing NOMA signals and for different optical powers, relating to wireless distances between $30 \mathrm{~m}$ and $100 \mathrm{~m}$.

\section{Acknowledgements}

This work was supported in part by the DFF FTP mmW-SPRAWL project, Spanish MINECO projects mUCORE (TEC2013-46917-C2-2-R) and SUNSET (TEC2014-59583-C2-1-R) co-funded by FEDER, Diputación General de Aragón (T25); Centro Universitario de la Defensa project BIO-DIVING (CUD2016-18) and MECD grant FPU-13/00620.

\section{References}

[1] T. Pfeiffer, "Next generation mobile fronthaul and midhaul architectures," IEEE J. Opt. Commun. Netw., Vol. 7, no. 11, pp. B38-B45, (2015).

[2] S. Rommel et al. "Outdoor W-band hybrid photonic wireless link based on an optical SFP+ module," Photon. Technol. Lett, Vol. 28, no. 21, pp. 2303-2306, (2016).

[3] K. Higuchi et al. "Non-orthogonal multiple access (NOMA) with successive interference cancellation for future radio access," IEICE Trans. Commun., Vol. E98-B, no. 3, pp. 403-414, (2015).

[4] M. I. Olmedo et al., "Multiband carrierless amplitude phase modulation for high capacity optical data links," J. Lightwave Technol., Vol. 32, no. 4, pp. 798-804, (2014).

[5] [21] ITU-T Recommendation G.975.1 (2004) 